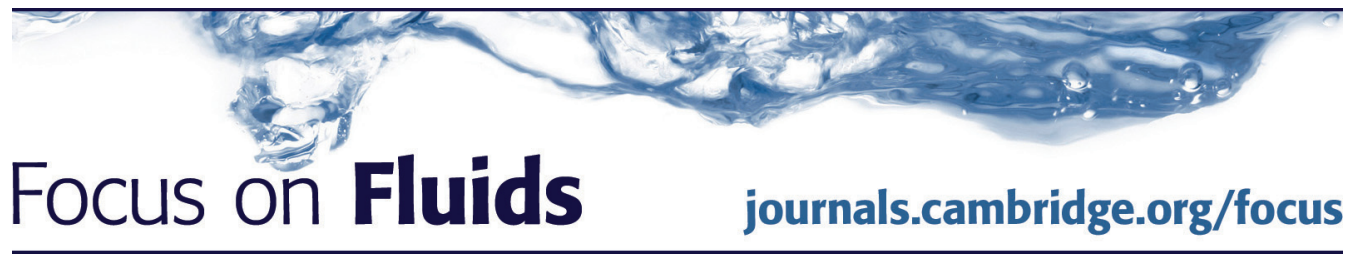

\title{
Meso-scale transport in sticky granular fluids
}

\section{S. Luding $\dagger$}

Multiscale Mechanics, Faculty of Engineering Technology, MESA+, University of Twente, P.O. Box 217, 7500AE Enschede, Netherlands

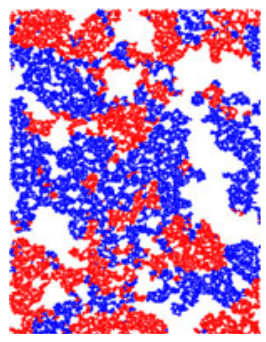

Fluid mechanics and rheology involve many unsolved challenges related to the transport mechanisms of mass, momentum and energy - especially when it comes to realistic, industrially relevant materials. Very interesting are suspensions or granular fluids with solid, particulate ingredients that feature contact mechanics on the micro-scale, which affect the transport properties on the continuum- or macro-scale. Their unique ability to behave as either fluid, or solid or both, can be quantified by non-Newtonian rheological rules, and results in interesting mechanisms such as super-diffusion, shear thickening, fluid-solid transitions (jamming) or relaxation/creep. Focusing on the steady state flow of a granular fluid, one can attempt to answer a long-standing question: how do realistic material properties such as dissipation, stiffness, friction or cohesion influence the rheology of a granular fluid? In a recent paper Macaulay \& Rognon (J. Fluid Mech., vol. 858, 2019, R2) shed new light on the effect cohesion can have on mass transport in sheared, sticky granular fluids. On top of the usual diffusive, stochastic modes of transport, cohesion can create and stabilise clusters of particles into bigger agglomerates that carry particles over large distances - either ballistically in the dilute regime, or by their rotation in the dense regime. Importantly, these clusters must not only be larger than the particles (defining the intermediate, meso-scale), but they must also have a finite lifetime, in order to be able to exchange mass with each other, which can seriously enhance transport in sticky granular fluids by rotection, i.e. a combination of rotation and convection.

Key words: granular media, non-continuum effects

\section{Introduction}

The macroscopic Navier-Stokes equations allow one to describe Newtonian fluids with constant transport coefficients (e.g. viscosity). Non-Newtonian systems, where the transport coefficients can depend on the state variables mass, momentum or energy densities, feature phenomena such as shear thickening, shear-band formation, clustering or plastic deformations related to creep/relaxation (Berger et al. 2015; Mari et al. 2015; Roy, Luding \& Weinhart 2017; Nicolas et al. 2018). How can we

\section{$†$ Email address for correspondence: s.luding@utwente.nl}


(a)

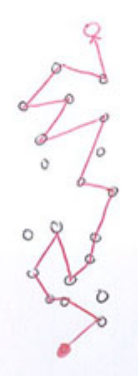

(b)

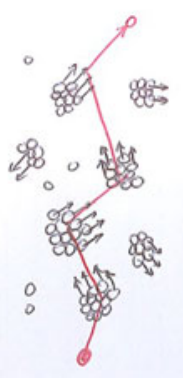

(c)

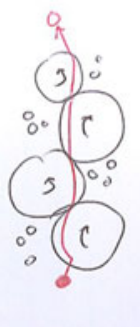

FIGURE 1. Sketch of three modes of mass transport: (a) diffusion, $(b)$ convection via clusters and (c) 'rotection', i.e. rotations of clusters mediating convection in dense shear flow as found by Macaulay \& Rognon (2019).

understand those phenomena that originate from the meso-scale, which is intermediate between atoms/particles and the hydrodynamic scale?

Each transport coefficient is related to the propagation and evolution of one (or more) of these quantities. For simple fluids (Hansen \& McDonald 1986), it is possible to bridge between the (macroscopic) hydrodynamic and the (microscopic) atomistic scales; as an example, the diffusion coefficient quantifies mass transport mediated by microscopic fluctuations, see figure 1(a). In the case of low density gases, the macroscopic equations and the transport coefficients can be obtained using the Boltzmann kinetic equation as a starting point. For moderate densities, the Enskog equation gives a good description of dense gases (or fluids) of hard atoms (Hansen \& McDonald 1986) or of particles including the effects of dissipation (Pöschel \& Luding 2001), reaching out (empirically) towards realistic systems (Luding 2009).

One interesting phenomenon caused by dissipation at low and moderate densities, occurring at a scale considerably larger than the particles, is the so-called clustering (hydrodynamic) instability (Luding 2009; Gonzalez, Thornton \& Luding 2014): in an initially homogeneous system, large-scale structures form in the presence of strong enough dissipation for a given system size and density, see figure $1(b)$. The associated large time of (free) motion between collisions of clusters, allows mass, momentum and energy to be transported much faster with the clusters, which changes mixing from diffusive to ballistic (https://www2.msm.ctw.utwente.nl/sluding/pictures/cooling.html).

Among many realistic material properties, friction typically enhances dissipation and thus clustering, whereas rotations of particles (due to frictional contacts) can help to randomise the system. Charges or cohesion are other practically relevant material properties: repulsive forces, with potential energy scale $\phi>0$, suppress structure formation since collisions become less probable, so that dissipation is reduced. In contrast, attractive forces, with cohesive (surface) energy $\phi<0$, can enhance structure formation by favouring collisions, enhancing dissipation and keeping particles together. Both forces can be unified into one framework and the onset of structure formation can be predicted by hydrodynamic stability analysis in a phase diagram with parameters dissipation and repulsion/cohesion (Gonzalez et al. 2014). The relevant dimensionless number is the relative strength of the interaction potential energy to the fluctuations of kinetic energy, $\Gamma=\phi / T_{g}$. The denominator, $T_{g}$, is the widely accepted 'granular temperature' that quantifies the microscopic fluctuations of kinetic energy, very much in analogy to the temperature in molecular systems. 
Cohesion becomes relevant for large enough $|\Gamma|$, which can occur either for strong interaction energy, $|\phi|$, or when $T_{g}$ is sufficiently small.

Since cluster-mediated mass transport requires some free space, one remaining question is: how is cohesion influencing the transport properties in very dense systems?

\section{Overview: sticky 'rotection'}

In their recent paper, Macaulay \& Rognon (2019) explain enhanced diffusion in rather dense, sheared, cohesive granular systems by large-scale structures (agglomerates) formed by sufficiently strong cohesion (Roy et al. 2017). In the absence of cohesion, clusters in sheared flows were reported already as early as 1986 (Drake 1990), but those have a rather short lifetime. Such meso-scale structures are considered as one of the major hurdles towards connecting the microscopic (particle) world with a purely macroscopic, hydrodynamic view on granular flows (Drake 1990).

The understanding of convective transport mediated by rotations of substructures in the system was first used as an explanation for the unusually strong heat transport in some geophysical systems (Griffani et al. 2013). Building on earlier work, Macaulay \& Rognon (2019) gain complementary insights from simulations on the convective mass transport mediated by the rotations of the intermediate-scale substructures. Their stochastic, meso-scale theory is an important piece of a bigger puzzle regarding the rich rheology in granular and other complex fluids.

How big are these cohesion induced clusters? And how long do they persist?

If the clusters have too short a lifetime, like in most cohesionless systems, they will not contribute significantly to the transport properties of the system and diffusion will remain mostly dominated by the particle size $d$ and their fluctuation velocity, so that the diffusion coefficient of the grains is $D_{T} \propto d v_{g}$, where $v_{g} \propto \sqrt{T_{g}}$. In contrast, when clusters are permanent, diffusion will be fully dominated by their size and fluctuation velocities, $v_{c}$, so that the diffusion coefficient of the clusters is $D_{c} \propto d_{c} v_{c}$, with corresponding 'cluster temperature' $v_{c} \propto \sqrt{T_{c}}$. Clusters, clumps or granules transport mass and momentum in one direction, but after a short while their direction of motion changes, giving rise to rather slow, random, diffusive transport on large time scales.

Macaulay and Rognon report a stochastic meso-scale model for the case of clusters having such a lifetime that they constructively contribute to transport (Macaulay \& Rognon 2019). In particular rotective transport of mass (convection by rotations) can be realised by particles rotating with a cluster (without displacement of the clusters themselves), see figure $1(c)$. However, in order to make this a ballistic, convective transport mode for particles, the clusters must give up their existence after a certain time, $\Psi$, so that these same particles can move further with a newly formed structure, in the same direction as before - and so on - over several cluster lifetimes.

This behaviour could be called super-diffusive, or turbulent-like, but one can also see it as convection through coherent handover and rotations of intermittent meso-scale structures in the flow. Macaulay \& Rognon (2019) demonstrate convection by rotation (i.e. rotection), with a diffusion coefficient $D \propto l_{c}^{2} \dot{\gamma}^{2} \Psi$, under shear rate $\dot{\gamma}$, for a wide range of cohesive strengths, if the cluster size, $l_{c}$, is correlated with a finite but limited lifetime, $\Psi$. After rotective transport over several cluster sizes, during several lifetimes, this new mode of transport becomes random and thus diffusive again. Open questions remaining are: what is the proper cluster temperature, $T_{c}$, and what is the control parameter replacing or complementing the energy ratio $\Gamma$ ? 


\section{Future: multi-scale fluids}

From the conceptual perspective, the present results by Macaulay \& Rognon (2019) deepen our understanding of multi-scale problems in general by considering also the fluctuations and physics at intermediate length scales. This might require an additional 'meso-scale temperature' that quantifies the fluctuation kinetics of meso-structures, clusters, clumps or agglomerates - involving their lifetimes, sizes and interactions, representing novel (possibly multiple) scale(s) in between the microand macro-scales.

In the presence of strong enough cohesion, the existence of enduring agglomerates is a fact used in wet granulation processes to make stable, bigger granules with much better processing and flow properties than the usually fine, sticky primary particles. Experimentally, the dependence of (dry) macroscopic cohesion on the particle size was recently studied (Shi et al. 2018) without reference to typical clusters and their sizes in such flows, since it was not possible to look inside the limestone powder used.

The new insights into the enhanced mass transport by rotection, i.e. convection enhanced by rotations in cohesive systems with meso-scale structures, are potentially relevant to understand and improve mixing in many industrial systems. The omnipresent segregation could then be counterbalanced by enhanced mixing triggered by the right amount of cohesion - neither too weak, nor too strong. This could reduce the costs of many products and improve the quality of materials and processes. But what is the right amount of cohesion? And how to keep cohesion that enhances mixing on the meso-scale under control is a practical problem yet unsolved.

\section{References}

Berger, N., Azema, E., Douce, J.-F. \& Radjai, F. 2015 Scaling behaviour of cohesive granular flows. Eur. Phys. Lett. 112 (6), 64004.

Drake, T. G. 1990 Structural features in granular flows. J. Geophys. Res. 95 (B6), 8681-8696.

Gonzalez, S., Thornton, A. R. \& Luding, S. 2014 Free cooling phase-diagram of hard-spheres with short- and long-range interactions. Eur. Phys. J. Spec. Top. 223 (11), 2205-2225.

Griffani, D., Rognon, P., Metzger, B. \& EinaV, I. 2013 How rotational vortices enhance transfers. Phys. Fluids 25 (9), 093301.

Hansen, J. P. \& MCDonald, I. R. 1986 Theory of Simple Liquids. Academic Press.

Luding, S. 2009 Towards dense, realistic granular media in 2D. Nonlinearity 22 (12), R101-R146.

Macaulay, M. \& Rognon, P. 2019 Shear-induced diffusion in cohesive granular flows: effect of enduring clusters. J. Fluid Mech. 858, R2.

Mari, R., Seto, R., Morris, J. F. \& Denn, M. M. 2015 Discontinuous shear thickening in Brownian suspensions by dynamic simulation. Proc. Natl Acad. Sci. 112 (50), 15326-15330.

Nicolas, A., Ferrero, E. E., Martens, K. \& Barrat, J.-L. 2018 Deformation and flow of amorphous solids: insights from elastoplastic models. Rev. Mod. Phys. 90 (4), 045006.

Pöschel, T. \& Luding, S. 2001 Granular Gases, vol. 564. Springer Science and Business Media.

RoY, S., Luding, S.\& WeINHART, T. 2017 A general(ized) local rheology for wet granular materials. New J. Phys. 19, 043014.

Shi, H., Mohanty, R., Chakravarty, S., Cabiscol, R., Morgeneyer, M., Zetzener, H., Ooi, J. Y., Kwade, A., Luding, S. \& Magnanimo, V. 2018 Effect of particle size and cohesion on powder yielding and flow. KONA Powder and Particle J. 35, 226-250. 